\title{
Dose Optimization in ${ }^{18}$ F-FDG PET Based on Noise-Equivalent Count Rate Measurement and Image Quality Assessment
}

\author{
Nivedita Rana*, Manpreet Kaur*, Harmandeep Singh, and Bhagwant Rai Mittal \\ Department of Nuclear Medicine, Post Graduate Institute of Medical Education and Research, Chandigarh, India
}

The present study aimed to optimize the injected dose of ${ }^{18} \mathrm{~F}$ FDG in whole-body PET/CT scans and assess its effect on noise-equivalent count rate (NECR) and visual image quality (IQ). Methods: Patients scheduled to undergo ${ }^{18} \mathrm{~F}-\mathrm{FDG} \mathrm{PET} /$ CT were prospectively recruited in the study from January to December 2019, regardless of the indication or underlying disease. Patients were divided into 4 groups and injected with different amounts of ${ }^{18} \mathrm{~F}-\mathrm{FDG}$ radioactivity per kilogram of body weight $(1.85,3.7,5.5$, and $7.4 \mathrm{MBq} / \mathrm{kg})$. All patients underwent ${ }^{18}$ F-FDG PET/CT studies, and NECR local $_{\text {lal }}$ was calculated by noting the trues rate, total prompts, and randoms rate for each bed position. Whole-body $\mathrm{NECR}_{\text {global }}$ was calculated as the average NECR for all bed positions. IQ was qualitatively assessed for each bed position ( $\left(\mathrm{Q}_{\mathrm{loca}}\right)$ and for whole-body PET ( $\left(\mathrm{Q}_{\mathrm{global}}\right)$ by 2 readers using 5-point scores based on prevalence of noise, contrast, and lesion detectability. NECR and IQ were compared among all 4 activity groups. Patients were also subdivided into 4 body-mass-index groups (group I, 15-20 kg/m²; group II, 20.1$25 \mathrm{~kg} / \mathrm{m}^{2}$; group III, $25.1-30 \mathrm{~kg} / \mathrm{m}^{2}$; and group IV, $30.1-35 \mathrm{~kg} / \mathrm{m}^{2}$ ) for comparison. A $P$ value of less than 0.05 was considered significant. Results: In total, 109 patients underwent ${ }^{18} \mathrm{~F}-\mathrm{FDG}$ PET/CT studies after injection of different amounts of ${ }^{18} \mathrm{~F}-\mathrm{FDG}$ radioactivity and a mean uptake time of $62.32 \mathrm{~min}$. The mean $\mathrm{NECR}_{\text {global }}$ and $I \mathrm{Q}_{\text {global }}$ for each group were significantly different from other groups $(P<0.05)$, with NECR and IQ being higher in high-activity groups than in low-activity groups. The overall IQ was acceptable in all patients, even in the lowest-activity group (1.84 $\mathrm{MBq} / \mathrm{kg}$ ). The mean $\mathrm{NECR}_{\text {global }}$ and $\mathrm{IQ}_{\text {global }}$ were significantly different in all 4 body-mass-index groups $(P<0.05)$, except between groups II and III $(P>0.05)$. NECR local $_{\text {lol }}$ and $\mathrm{IQ}_{\text {local }}$ correlated moderately $(r=0.64)$. Conclusion: Optimization of injected ${ }^{18} \mathrm{~F}-\mathrm{FDG}$ radioactivity from $7.4 \mathrm{MBq} / \mathrm{kg}(200 \mu \mathrm{Ci} / \mathrm{kg})$ to $1.85 \mathrm{MBq} / \mathrm{kg}(50 \mu \mathrm{Ci} / \mathrm{kg})$ resulted in acceptable IQ, despite a reduction in NECR.

Key Words: NECR; image quality; FDG PET; dose optimization J Nucl Med Technol 2021; 49:49-53 DOI: $10.2967 /$ jnmt.120.250282

\footnotetext{
Received May 26, 2020; revision accepted Jul. 22, 2020.

For correspondence or reprints contact: Harmandeep Singh, Department of Nuclear Medicine, PGIMER, Chandigarh 160012, India.

E-mail: drharmandeepsingh@gmail.com

${ }^{*}$ Contributed equally to this work.

Published online Sep. 4, 2020.

COPYRIGHT (c) 2021 by the Society of Nuclear Medicine and Molecular Imaging.
}

$\mathbf{T}$ he burgeoning demand for PET/CT imaging in providing personalized health care has led to a continuous evolution of technology to provide the best image quality (IQ). This evolution has resulted in incorporation of advances such as time-of-flight and point-spread function in reconstruction algorithms to improve the spatial resolution of the PET images (1-4). PET/CT scanners that include time-of-flight and point-spread function aim to get good IQ even with less radioactivity injected into the patient $(5,6)$. Decreasing the dose of injected activity is always a welcome step to reduce exposure of the patient to radiation. However, despite the use of advanced technology, there is a limit below which the administered radioactivity cannot be reduced without increasing the acquisition time to get good IQ. Therefore, the injected dose and acquisition time need to be balanced to achieve diagnostic PET IQ.

PET IQ assessment is an arduous task because of the physics of PET image acquisition. Not all the counts acquired during a PET acquisition are true coincidence counts; random and scatter counts are also included. These random and scatter counts do not reflect the true activity distribution in the body and, thus, deteriorate the PET IQ. Using signal-to-noise ratio as a quantifying parameter for IQ assessment is not very promising, since it takes into account all the prompt counts, including random and scatter counts. However, noise-equivalent count rate (NECR) is the IQ parameter that quantifies the contribution of true counts in total prompts and thus is more representative of IQ. A higher NECR ensures a better signal-to-noise ratio in the patient data $(6,7)$.

NECR has been used routinely as a parameter for comparing the IQ of PET scanners. Its use as a tool for assessing IQ in clinical PET/CT images has been reported for a few years (69). There is limited literature on the value of NECR in relation to activity injected per kilogram of body weight and its relation to IQ. The studies computing NECR as an IQ parameter are mostly phantom or simulation studies (10-12). Two retrospective studies have measured NECR in patient populations, and one of these studies has calculated only the regional NECR of the liver (8-9).

According to European Association of Nuclear Medicine guidelines, activity is administered linearly or quadratically with patient weight and acquisition time (13). Keeping 
a minimum acquisition time of $1 \mathrm{~min}$, and for a patient weight of $45-75 \mathrm{~kg}$, the ${ }^{18} \mathrm{~F}-\mathrm{FDG}$ dose ranges from 4.07 to $7.03 \mathrm{MBq} / \mathrm{kg}(110-190 \mu \mathrm{Ci} / \mathrm{kg})$. Other studies have also recommended doses ranging from 5.55 to $7.4 \mathrm{MBq} / \mathrm{kg}$ (150-200 $\mu \mathrm{Ci} / \mathrm{kg})(9-10,14)$.

The present study aimed to optimize the injected dose of ${ }^{18}$ F-FDG to reduce the effective exposure from whole-body PET/CT scans and to assess the effect of this optimization on NECR and visual IQ assessment.

\section{MATERIALS AND METHODS}

\section{Patient Population}

This prospective study included 112 patients who were referred for ${ }^{18}$ F-FDG PET/CT imaging from January to December 2019, regardless of indication. The study was approved by the departmental review board, and written informed consent was obtained from all patients. Those with fasting glucose levels higher than $200 \mathrm{mg} /$ $\mathrm{dL}$ and those who had not fasted for at least $4 \mathrm{~h}$ were not included. Patients for whom partial extravasation of the ${ }^{18} \mathrm{~F}-\mathrm{FDG}$ activity was seen on the PET/CT images were excluded, as extravasation affects the quantification of IQ. The patients were divided into 4 groups (groups A-D) depending on the amount of ${ }^{18} \mathrm{~F}-\mathrm{FDG}$ radioactivity injected per kilogram of body weight. In groups A, B, C, and D, the injected dose was $1.85 \mathrm{MBq} / \mathrm{kg}(50 \mu \mathrm{Ci} / \mathrm{kg}), 3.7 \mathrm{MBq} / \mathrm{kg}(100 \mu \mathrm{Ci} /$ $\mathrm{kg}), 5.5 \mathrm{MBq} / \mathrm{kg}(150 \mu \mathrm{Ci} / \mathrm{kg})$, and $7.4 \mathrm{MBq} / \mathrm{kg}(200 \mu \mathrm{Ci} / \mathrm{kg})$, respectively. Further, the study population was divided into 4 groups (groups I-IV) based on body mass index (BMI) for comparison of the effect of BMI on IQ and NECR. Groups I, II, III, and IV had a BMI of $15-20,20.1-25,25.1-30$, and $30.1-35 \mathrm{~kg} / \mathrm{m}^{2}$, respectively.

\section{PET/CT Imaging}

All patients underwent whole-body PET/CT from the base of the skull to the mid thigh using a 3-dimensional time-of-flight-based scanner (Discovery MIDR; GE Healthcare) at 45-75 min after administration of the radioactivity. A helical CT scan using a tube voltage of 120 $\mathrm{kVp}$ and a variable tube current $(150-350 \mathrm{~mA})$ was acquired craniocaudally, followed by a PET scan in the caudocranial direction, at a rate of $90 \mathrm{~s}$ per bed position for group A and $60 \mathrm{~s}$ for the remaining 3 groups. The whole-body PET images were reconstructed in a matrix of $192 \times 192$ using ordered-subset expectation maximization (24 subsets, 2 iterations) and a $z$-axis gaussian filter of $5.5 \mathrm{~mm}$ in full width at half maximum. During the PET acquisition, counts (true counts, total prompts, and randoms) were recorded for each bed position.

\section{Image Analysis}

PET IQ was assessed using 2 methods: quantitative and qualitative. Quantitative IQ was assessed by measuring NECR for each bed position $\left(\mathrm{NECR}_{\text {local }}\right)$. This measurement was done by taking the ratio of the square of true counts to the sum of true counts, randoms, and scatter counts (total prompts). The unit for NECR was kilocounts per second (kcps). The NECR for the whole-body scan of a patient $\left(\mathrm{NECR}_{\text {global }}\right)$ was defined as the mean $\mathrm{NECR}_{\text {local }}$ for all bed positions in a whole-body acquisition.

For the clinical and qualitative IQ analysis, the PET/CT images were transferred to a dedicated review workstation (Advantage Workstation 4.7; GE Healthcare). PET maximum-intensity-projection and transaxial IQ was visually assessed and scored by a nuclear medicine physician and a physicist, each having more than 8 y of experience. The IQ local score ( $\left.\mathrm{IQ}_{\text {local }}\right)$ was a 5-point scale assigned to each bed position, where 1 is poor, 2 is bad, 3 is acceptable/average, 4 is good, and 5 is excellent. The IQ global score $\left(\mathrm{IQ}_{\mathrm{global}}\right)$, which used the same 5-point scale, was assigned to the whole study after assessment of all bed positions and maximum-intensity-projection images. The readers assessed PET IQ subjectively on the basis of prevalence of noise, contrast between different tissues and organs, and lesion detectability.

\section{Statistical Analysis}

Quantitative parameters with a normal distribution were expressed using mean and SD. The mean $\mathrm{NECR}_{\text {global }}$ and $\mathrm{IQ}_{\text {global }}$ were compared in all 4 groups using independent $t$ testing for each pair of groups. The mean $\mathrm{NECR}_{\text {global }}$ and $\mathrm{IQ}_{\text {global }}$ were compared in the 4 BMI groups using independent $t$ testing for each pair of groups. The correlation among $\mathrm{NECR}_{\text {global }}, \mathrm{IQ}_{\text {global }}$, and BMI, as well as among $\mathrm{NECR}_{\text {local }}, \mathrm{IQ}_{\text {local }}$, and BMI, was assessed using the Pearson correlation coefficient. The 2-way multivariate analysis was applied to study to see if there was any interaction between the activity group and the BMI group while evaluating $\mathrm{NECR}_{\text {global }}$ and $\mathrm{IQ}_{\text {global }}$. $P$ values were considered significant if they were less than 0.05 .

\section{RESULTS}

In total, 112 patients were recruited, of whom 3 were excluded because of partial extravasation of radioactivity. Only 109 patients (38 men, 71 women) in the age group (14-80 y) with a mean age of $49.8 \pm 16.1 \mathrm{y}$ were included. Descriptive mean values of injected activity, $\mathrm{NECR}_{\text {global }}$, $\mathrm{IQ}_{\mathrm{global}}, \mathrm{BMI}$, and uptake time in groups $\mathrm{A}(n=18), \mathrm{B}$ $(n=30), \mathrm{C}(n=32)$, and $\mathrm{D}(n=29)$ have been tabulated in Supplemental Table 1 (supplemental materials are available at http://jnm.snmjournals.org).

\section{Comparison of $\mathbf{N E C R}_{\text {global }}$ and $\mathbf{I Q}_{\text {global }}$ in Different Activity Groups}

$\mathrm{NECR}_{\text {global }}$ and $\mathrm{IQ}_{\mathrm{global}}$ showed a statistically significant difference $(P<0.05)$ among all 4 activity groups, with group D showing highest $\mathrm{NECR}_{\text {global }}$ and $\mathrm{IQ}_{\text {global }}$ scores and group $\mathrm{A}$ showing the lowest $\mathrm{NECR}_{\text {global }}$ and $\mathrm{IQ}_{\text {global }}$ scores. The mean $\mathrm{IQ}_{\text {global }}$ score in group A was 3, denoting an overall acceptable IQ, even in the lowest-activity group. No study was reported as having bad or poor IQ in any group.

\section{Correlation of IQ Scores and BMI}

The correlation coefficient between $\mathrm{NECR}_{\text {global }}$ and $\mathrm{IQ}_{\text {global }}$ was $0.47(P<0.05)$, and the correlation of NEC$\mathrm{R}_{\text {global }}$ and $\mathrm{IQ}_{\mathrm{global}}$ with BMI was negative, at $r=-0.46$ and $-0.40(P<0.05)$, respectively. $\mathrm{NECR}_{\text {local }}$ and $\mathrm{IQ}_{\text {local }}$ were moderately correlated with each other, having a correlation coefficient of $r=0.64(P<0.05)$. No significant correlation was found between $\mathrm{NECR}_{\text {local }}$ and BMI or between $\mathrm{IQ}_{\text {local }}$ score and BMI (Fig. 1).

\section{Comparison of $\mathrm{NECR}_{\text {global }}$ and $I Q_{\text {global }}$ in Different BMI Groups}

The variation in mean $\mathrm{NECR}_{\text {global }}$ and $\mathrm{IQ}_{\text {global }}$ for the different BMI groups within each activity group is shown in Supplemental Figure 1. The mean $\mathrm{NECR}_{\text {global }}$ and $\mathrm{IQ}_{\text {global }}$ were significantly different in all 4 BMI groups $(P<0.05)$, except between groups II and III $(P>0.05)$. 


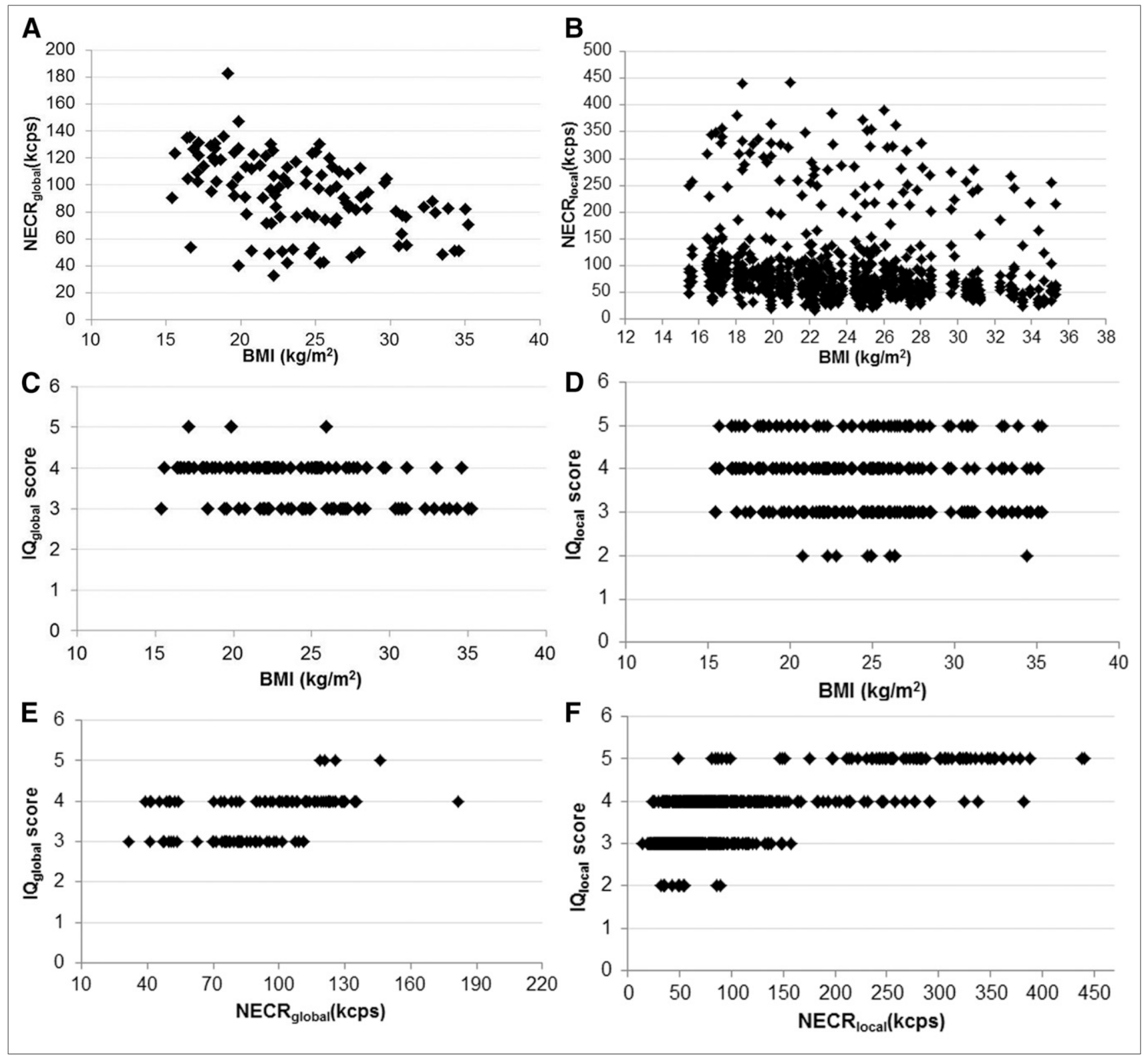

FIGURE 1. Variation in value of $N E C R_{\text {global }}$ with $B M I(A), N E C R_{\text {local }}$ with $B M I(B), Q_{\text {global }}$ score with $B M I(C), I Q_{\text {local }} S c o r e$ with $B M I$ (D), IQ $Q_{\text {global }}$ score with $N E C R_{\text {global }}(E)$, and IQlocal score with $\mathrm{NECR}_{\text {local }}(\mathrm{F})$.

The difference in visual IQ in patients with similar BMIs but injected with different amounts of radioactivity can be seen in Figures 2 and 3.

\section{Interaction Effect Between Activity Group and BMI Group on IQ Parameters}

The 2-way multivariate analysis showed no statistically significant interaction effect between activity group and BMI group on either $\mathrm{NECR}_{\text {global }}$ or $\mathrm{IQ}_{\text {global }}\left(F_{12,190}=1.631\right.$, $P=0.086$; Wilks $\lambda=0.822$ ).

\section{DISCUSSION}

In any type of nuclear medicine procedure, dose optimization is a major concern because it aims to minimize exposure of patients and staff to radiation while preserving
IQ. The amount of activity administered to the patient can be reduced according to the as-low-as-reasonably-achievable principle. However, a reduction in administered activity can compromise IQ if the resultant counts are insufficient. An increase in administered activity in PET (beyond a certain level) also degrades IQ, since random counts also increase. Optimization of the administered dose and IQ is essential for ${ }^{18}$ F-FDG PET/CT studies. The impetus behind the rapid evolution of PET instrumentation and reconstruction algorithms is to minimize the activity injected into patients as much as possible while maintaining diagnostic IQ. Use of improved scintillator crystals such as lutetium-yttrium oxyorthosilicate and incorporation of techniques such as time-offlight and point-spread function in reconstruction algorithms 


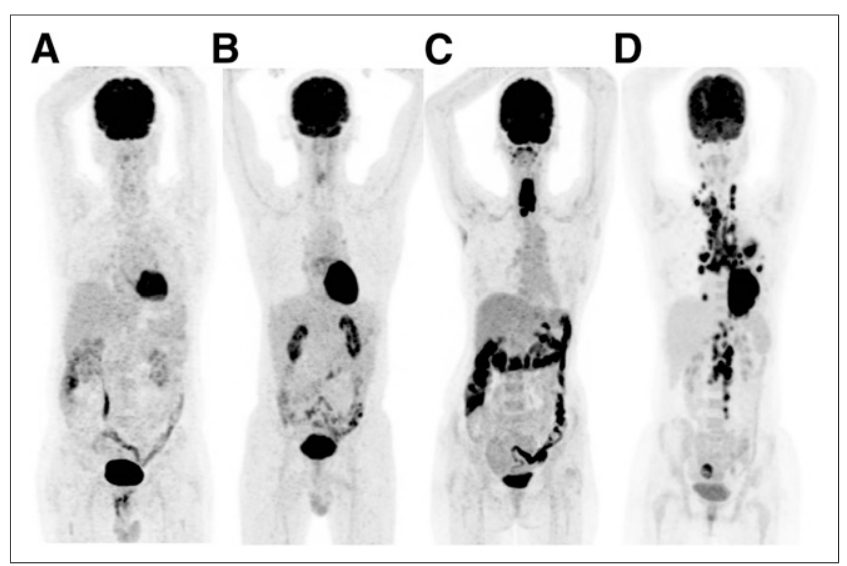

FIGURE 2. ${ }^{18}$ F-FDG PET maximum-intensity-projection images of 4 different patients having approximately the same BMI $\left(20.00 \mathrm{~kg} / \mathrm{m}^{2}\right)$. (A) Administered activity: $1.85 \mathrm{MBq} / \mathrm{kg}$ (50 $\mathrm{MCi} / \mathrm{kg}) ; N^{2} \mathrm{NCR}_{\text {global }}, 50.55 \mathrm{kcps}$; IQ global score, 3. (B) Administered activity: $3.7 \mathrm{MBq} / \mathrm{kg}(100 \mu \mathrm{Ci} / \mathrm{kg})$; NECR $\mathrm{Nlobal}_{\text {, }}$ $77.78 \mathrm{kcps}$; IQ global score, 4. (C) Administered activity: 5.5 $\mathrm{MBq} / \mathrm{kg}(150 \mu \mathrm{Ci} / \mathrm{kg}) ; \mathrm{NECR}_{\text {global }}, 90.23 \mathrm{kcps}$; IQ $\mathrm{Q}_{\text {global }}$ score, 4. (D) Administered activity: $7.4 \mathrm{MBq} / \mathrm{kg}(200 \mu \mathrm{Ci} / \mathrm{kg}) ;$ NECR global, $_{146.30}$ kcps; $I_{\text {global }}$ score, 5.

have made this minimization feasible by improving spatial resolution, increasing image contrast, and decreasing noise in the image.

NECR has been used as a quantitative PET IQ parameter either in phantom studies or in simulation studies (10-12). Only a few studies have used it as an analysis parameter in patient studies. Chang et al. studied the effect of injected dose and BMI on NECR, but they computed NECR for only 1 bed position, with liver (8). Queiroz et al. also assessed clinical IQ and compared it with NECR measurements in a

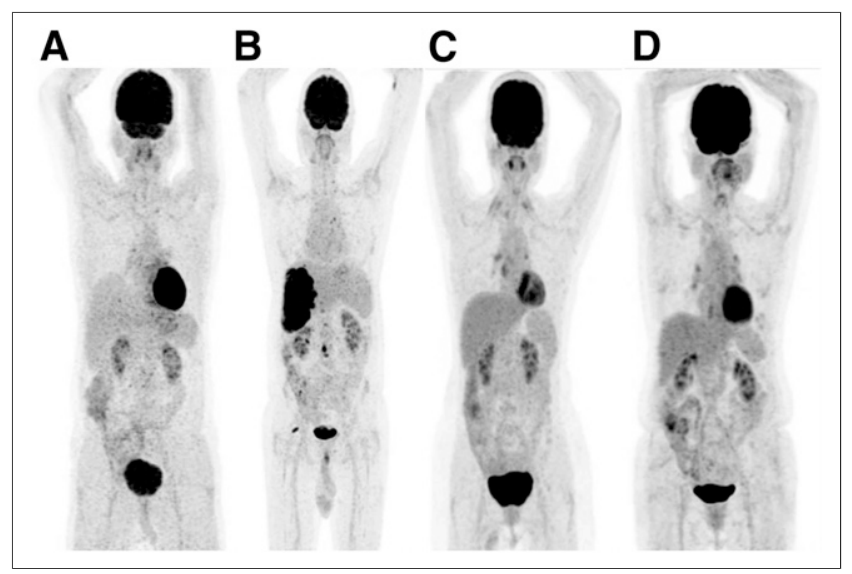

FIGURE 3. ${ }^{18} \mathrm{~F}-\mathrm{FDG}$ PET maximum-intensity-projection images of 4 different patients having approximately the same BMI $\left(25.00 \mathrm{~kg} / \mathrm{m}^{2}\right)$. (A) Administered activity, $1.85 \mathrm{MBq} / \mathrm{kg}(50$ $\mu \mathrm{Ci} / \mathrm{kg}$ ); NECR global, $48.25 \mathrm{kcps}$; IQ $\mathrm{Q}_{\text {global }}$ score, 3. (B) Administered activity, 3.7 MBq/kg (100 $\mu \mathrm{Ci} / \mathrm{kg})$; NECR ${ }_{\text {global }}, 75.72 \mathrm{kcps}$; IQ $\mathrm{Q}_{\text {global }}$ score, 3. (C) Administered activity, $5.5 \mathrm{MBq} / \mathrm{kg}(150 \mu \mathrm{Ci} / \mathrm{kg})$;

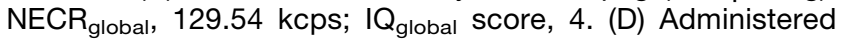
activity, 7.4 MBq/kg (200 $\mu \mathrm{Ci} / \mathrm{kg}) ; \mathrm{NECR}_{\text {global }}, 122.94 \mathrm{kcps}$; $\mathrm{IQ}_{\text {global }}$ score, 4. patient population (9). NECR can be used as an objective measure of the IQ of a PET system. A higher NECR is expected to ensure a good signal-to-noise ratio and less noise in the reconstructed images. In the present study, NECR along with visual IQ assessment was used to assess IQ for ${ }^{18}$ F-FDG PET images acquired using different weight-based doses.

The mean $\mathrm{NECR}_{\text {global }}$ for all patients $(n=109)$ in our study was $92.57 \mathrm{kcps}$. This is lower than the mean $\mathrm{NECR}_{\text {global }}$ computed by Queiroz et al. in their study ( $n=75$, NECR $106.4 \mathrm{kcps})$ (9). Also, the mean NECR $\mathrm{global}_{\text {calculated in }}$ patients segregated according to BMI was 91.5, 85.25, 88.5, and $63.22 \mathrm{kcps}$ in groups I, II, III, and IV, respectively, in the present study, as compared with 133.04, 112.49, 102.34, and $86.79 \mathrm{kcps}$, respectively, in their study. The decreased mean value in the present study can be explained by inclusion of an even lower-activity group of $1.85 \mathrm{MBq} / \mathrm{kg}(50 \mu \mathrm{Ci} / \mathrm{kg})$ in our study. A trend for NECR to increase with decreasing BMI was observed in our study, except for groups II and III, for which there was no statistically significant difference in the mean values. A similar trend was observed in the study by Queiroz et al. The value of NECR calculated in the present study cannot be compared with that computed in the study by Chang et al., as they calculated the NECR for only 1 bed position, liver, whereas we calculated it for each bed position and then averaged the values (8).

Though many studies have compared NECR with visual assessment, no study, to our knowledge, has compared NECR and visual assessment in patient groups injected with different amounts of activity per kilogram of body weight. The quantitative and qualitative IQ analysis in our study showed a significant difference in patient groups injected with different activities. The mean $\mathrm{NECR}_{\text {global }}$ and $\mathrm{IQ}_{\text {global }}$ were highest for the group injected with $7.4 \mathrm{MBq} /$ $\mathrm{kg}(200 \mu \mathrm{Ci} / \mathrm{kg})$ and lowest for the group injected with 1.85 $\mathrm{MBq} / \mathrm{kg}(50 \mu \mathrm{Ci} / \mathrm{kg})$, as shown in Supplemental Table 1. However, in a similar study, Chang et al. compared the local NECR for liver and found no statistically significant change when the injected activity was increased from 296$444 \mathrm{MBq}(8-12 \mathrm{mCi})$ to $555-740 \mathrm{MBq}(15-20 \mathrm{mCi})(8)$. The difference in findings between the 2 studies can be explained by use of a higher range of activities in their study than in ours (89.91-463.24 MBq [2.43-12.52 mCi]).

Queiroz et al. showed that patient NECR and the respective IQ correlated strongly with each other and negatively with BMI (9). They also found a significant positive correlation between $\mathrm{IQ}_{\text {local }}$ and $\mathrm{NECR}_{\text {local. }}$ A similar moderately significant correlation between $\mathrm{IQ}_{\text {local }}$ and $\mathrm{NECR}_{\text {local }}$ was observed in the present study. $\mathrm{NECR}_{\text {global }}$ also correlated with $\mathrm{IQ}_{\text {global }}$, and both these parameters had a negative correlation with BMI; however, the correlation was weak. One possible explanation can be use of a discrete score in our study, unlike the continuous score used by Queiroz et al. (9).

Several studies have been done to optimize ${ }^{18} \mathrm{~F}-\mathrm{FDG}$ activity in order to achieve good IQ. In a study by Geismar et al., the authors concluded that an optimal ${ }^{18}$ F-FDG dose 
of $4 \mathrm{MBq} / \mathrm{kg}$ can be used only in patients with a BMI of 22 $\mathrm{kg} / \mathrm{m}^{2}$ or less; a dose of $5 \mathrm{MBq} / \mathrm{kg}$ was needed to obtain good IQ in patients with a BMI of more than $22 \mathrm{~kg} / \mathrm{m}^{2}(15)$. A similar study by Everaert et al. determined an ${ }^{18} \mathrm{~F}-\mathrm{FDG}$ activity of at least $8 \mathrm{MBq} / \mathrm{kg}(200 \mu \mathrm{Ci} / \mathrm{kg})$ to be an optimized dose, at 2-3 min per bed position, for obtaining good IQ for lutetium oxyorthosilicate PET/CT scanners (14). However, in the present study, in the group with the second lowest activity - that is, $3.7 \mathrm{MBq} / \mathrm{kg}$ - as well as for the highest-BMI patient $\left(35.28 \mathrm{~kg} / \mathrm{m}^{2}\right)$, and with an acquisition time of 1 min per bed position, diagnostic IQ was maintained. A comparable optimized dose of $3.8 \mathrm{MBq} / \mathrm{kg}$ has been given by Queiroz et al., but their study was retrospective and not supported by any patient images (9). The feasibility of reducing the injected activity to $1.85 \mathrm{MBq} / \mathrm{kg}(50 \mu \mathrm{Ci} / \mathrm{kg})$ was investigated in a group of 18 patients by increasing the acquisition time from $60 \mathrm{~s}$ to $90 \mathrm{~s}$ per bed position. Though the NECR was comparatively reduced, acceptable diagnostic IQ could be achieved in this group as well, even for patients having a BMI of as high as $34.64 \mathrm{~kg} / \mathrm{m}^{2}$.

For all 109 patients in the present study, with an administered activity from 1.85 to $7.4 \mathrm{MBq} / \mathrm{kg}$ body in different groups, the IQ score was at least 3. This implies that the IQ was acceptable to excellent in patients with different BMIs. An ${ }^{18}$ F-FDG activity of at least $1.85 \mathrm{MBq} / \mathrm{kg}$ was considered optimal for obtaining acceptable IQ in patients undergoing whole-body ${ }^{18} \mathrm{~F}-\mathrm{FDG}$ PET/CT using an imaging time of 60-90 s per bed position, depending on the administered activity. In the future, PET scanners with even faster crystals, coupled with silicon-based photomultiplier tubes aiming to enhance system sensitivity and spatial resolution, can pave the way for use of an even lower administered radioactivity.

\section{CONCLUSION}

Optimization of the injected ${ }^{18} \mathrm{~F}-\mathrm{FDG}$ radioactivity from $7.4 \mathrm{MBq} / \mathrm{kg}(200 \mu \mathrm{Ci} / \mathrm{kg})$ to $1.85 \mathrm{MBq} / \mathrm{kg}(50 \mu \mathrm{Ci} / \mathrm{kg})$ resulted in excellent to acceptable IQ in all patients. Administration of a low radiotracer activity $(1.85 \mathrm{MBq} / \mathrm{kg})$ can achieve an acceptable PET IQ while reducing exposure of patients to radiation.

\section{DISCLOSURE}

No potential conflict of interest relevant to this article was reported.

\section{REFERENCES}

1. Lois C, Jakoby BW, Long MJ, et al. An assessment of the impact of incorporating time-of-flight information into clinical PET/CT imaging. J Nucl Med. 2010; 51:237-245.

2. Surti S, Karp JS. Experimental evaluation of a simple lesion detection task with time-of-flight PET. Phys Med Biol. 2009;54:373-384.

3. Martí-Climent JM, Prieto E, Dominguez-Prado I, et al. Contribution of time of flight and point spread function modeling to the performance characteristics of the PET/CT Biograph mCT scanner. Rev Esp Med Nucl Imagen Mol. 2013;32: 13-21.

4. Panin VY, Kehren F, Michel C, Casey M. Fully 3-D PET reconstruction with system matrix derived from point source measurements. IEEE Trans Med Imaging. 2006;25:907-921.

5. Taniguchi T, Akamatsu G, Kasahara Y, et al. Improvement in PET/CT image quality in overweight patients with PSF and TOF. Ann Nucl Med. 2015;29:7177.

6. Reynés-Llompart G, Sabaté-Llobera A, Llinares-Tello E, Martí-Climent JM, Gámez-Cenzano C. Image quality evaluation in a modern PET system: impact of new reconstructions methods and a radiomics approach. Sci Rep. 2019; 9:10640.

7. Karakatsanis NA, Fokou E, Tsoumpas C. Dosage optimization in positron emission tomography: state-of-the-art methods and future prospects. Am J Nucl Med Mol Imaging. 2015;5:527-547.

8. Chang T, Chang G, Kohlmyer S, Clark JW, Rohren E, Mawlawi OR. Effects of injected dose, BMI and scanner type on NECR and image noise in PET imaging. Phys Med Biol. 2011;56:5275-5285.

9. Queiroz MA, Wollenweber SD, Von Schulthess G, Delso G, Veit-Haibach P. Clinical image quality perception and its relation to NECR measurements in PET. EJNMMI Phys. 2014;1:103.

10. Watson CC, Casey ME, Bendriem B, et al. Optimizing injected dose in clinical PET by accurately modeling the counting-rate response functions specific to individual patient scans. $J$ Nucl Med. 2005;46:1825-1834.

11. Lartizien C, Comtat C, Kinahan PE, Ferreira N, Bendriem B, Trébossen R. Optimization of injected dose based on noise equivalent count rates for 2- and 3-dimensional whole-body PET. J Nucl Med. 2002;43:1268-1278.

12. Danna M, Lecchi M, Bettinardi V, et al. Generation of the acquisition-specific NEC (AS-NEC) curves to optimize the injected dose in 3D ${ }^{18} \mathrm{~F}-\mathrm{FDG}$ whole body PET studies. IEEE Trans Nucl Sci. 2006;53:86-92.

13. Boellaard R, O'Doherty MJ, Weber W, et al. FDG PET and PET/CT: EANM procedure guidelines for tumour PET imaging-version 1.0. Eur J Nucl Med Mol Imaging. 2010;37:181-200.

14. Everaert H, Vanhove C, Lahoutte T, et al. Optimal dose of ${ }^{18} \mathrm{~F}-\mathrm{FDG}$ required for whole-body PET using an LSO PET camera. Eur J Nucl Med Mol Imaging. 2003;30:1615-1619.

15. Geismar JH, Stolzmann P, Sah B, Burger IA, Seifert B. Intra-individual comparison of PET/CT with different body weight-adapted FDG dosage regimens. Acta Radiol Open. 2015;4:2047981614560076. 